\title{
Schlussbetrachtung
}





\section{Die Großregion SaarLorLux: Lebenswirklichkeiten und politische Konstruktionen}

BiRTE NiENABER

Was ist die Großregion SaarLorLux? Handelt es sich um eine oder mehrere Lebenswirklichkeiten? Um eine oder mehrere politische Konstruktionen? Oder: Ist die Großregion SaarLorLux vielleicht etwas ganz anderes? Mit diesen Fragen befasste sich dieser Sammelband. Am Ende des Bandes angekommen gilt es nun, Antworten zu geben oder zumindest den Versuch zu unternehmen, Antworten zu finden. Dies mag auf den ersten Blick leichter klingen, als es letztendlich ist.

Der Begriff >Großregion< verweist zunächst auf keine in einem Schulatlas aufzufindende Region. Der Begriff lässt höchstens Assoziationen zu wie >großflächig<, >großartig< oder >größenwahnsinnigく, aber keine Identifikation von einem Raum. Erst durch den Zusatz >SaarLorLux< kommt eine räumliche Dimension ins Spiel, die jedoch - wie in diesem Band mehrfach erläutert - nicht die gesamte Großregion SaarLorLux umfasst. Die in dem Kürzel >SaarLorLux< abwesenden Teilregionen Wallonien und Rheinland-Pfalz bleiben außen vor, da sie in die Anfänge der grenzüberschreitenden Zusammenarbeit noch nicht einbezogen waren.

Berücksichtigt man nun die Beiträge dieses Bandes und versucht der Frage nach den Lebenswirklichkeiten und politischen Konstruktionen in der Großregion SaarLorLux nachzugehen, ergeben sich verschiedene Sichtweisen. Zunächst gibt es Beiträge, die die ökonomische, soziale oder kulturelle Lebenswirklichkeit der Großregion SaarLorLux hervorheben (z. B. Dörrenbächer, Helfer, Belkacem/Pigeron-Piroth, Wille, Boesen/Schnuer und Kreutzer) und zeigen, dass solche Lebenswirklichkeiten unterschiedlich ausgeprägt sein können - z. B. stärker in Grenznähe, schwächer in Grenzferne oder nur für bestimmte Bevölkerungsgruppen und Akteure. Diese Beiträge zeigen deutlich, dass es nicht >eine< Lebenswirklichkeit gibt, sondern dass sie - im Plural - vom jeweiligen Untersuchungsobjekt und Individuum abhängen. Ferner können 
sich Lebenswirklichkeiten über die Zeit verändern; während seit vielen Jahrhunderten bereits die ökonomische Integration in der Großregion SaarLorLux zu einer Lebenswirklichkeit von Menschen und Unternehmen geworden ist, sind andere Aspekte (z. B. sozio-kulturelle) noch relativ jung.

Aus einem anderen Blickwinkel werden in diesem Band eher politische Konstruktionen betont (z. B. Clément und Evrard/Schulz). Die Beiträge dieser Gruppe gehen v. a. auf die Formen der politisch-administrativen und raumplanerischen Institutionalisierungen der Großregion SaarLorLux in ihren vielfältigen Erscheinungsformen ein. Dabei treten der unterschiedliche Wille der einzelnen Teilregionen zur Integration sowie ihre unterschiedlichen Kompetenzen und damit ihre politisch-rechtlichen Instrumentarien deutlich in den Vordergrund. Es wird veranschaulicht, dass die räumliche Dimension der heutigen Großregion SaarLorLux aufgrund politisch-institutioneller Notwendigkeiten entstand, d. h. konstruiert wurde.

Des Weiteren werden in den Buchbeiträgen politische Konstruktionen und Lebenswirklichkeiten in einen Zusammenhang gebracht und ihr Wechselverhältnis herausgestellt (z. B. Schönwald, Scholz, Goulet/Vatter, Sonntag, Crenn und Mendgen). Bei den Autoren, die diese Zusammenhänge darlegen, zeigt sich, dass ohne bereits existierende grenzüberschreitende Lebenswirklichkeiten keine politischen Konstruktionen (z. B. im Medienbereich oder im Kultursektor) stattfänden; umgekehrt aber die politische Konstruktion >Großregion SaarLorLux a auch als Grundlage für Identitätsbildung oder Kulturförderung dient. Außerdem wird in einigen Beiträgen darauf verwiesen, dass die Lebenswirklichkeiten eher entlang der Grenzen stattfinden, die politischen Konstruktionen hingegen eher im gesamten Territorium der Großregion SaarLorLux.

Eine eindeutige Antwort auf die Frage, ob die Großregion SaarLorLux eine Lebenswirklichkeit oder eine politische Konstruktion ist, lässt sich - wie dieser Band gezeigt hat - kaum geben. Die Beiträge zeigen hingegen deutlich: Die Großregion SaarLorLux eint v. a. ihre Heterogenität. So existieren unterschiedliche politische und individuelle Partikularinteressen, unterschiedliche Subventions-, Rechts-, Raumplanungs-, Bildungs-, Kultur-, Migration-, Mobilitäts-, Wirtschafts- oder auch Sprachmuster nebeneinander. Sie sind teilweise an territoriale Grenzen gebunden, zum Teil aber überschreiten sie diese auch und stellen neue beispielsweise mentale, materielle oder kulturelle Grenzen her. Solche multiplen Grenzziehungen wirken sowohl auf die Außenabgrenzung der Großregion SaarLorLux als auch innerhalb derselben. Wie diese Grenzziehungen geschehen, hängt mit den Lebenswirklichkeiten und den verschiedenen politischen Konstruktionen jedes einzelnen Akteurs, Entscheidungsträgers und Bürgers in der Großregion SaarLorLux zusammen. So können »Kernbereich« und »Randgebiete« (Schönwald in diesem Band), variable Geometrien (vgl. Evrard/Schulz in diesem Band), »Regionen mehrerer Geschwindigkeiten« (Sonntag in diesem Band), »unterschiedliche Aktionsradien« (Scholz in diesem 
Band) sowie vielfältige politische Gremien und Institutionalisierungsgrade (vgl. Clément in diesem Band) zu multiplen Grenzziehungen beitragen.

Grenzenüberwindend wirken in der Großregion SaarLorLux besonders ökonomische Faktoren. Der Arbeitsmarkt lässt viele Grenzpendler täglich v.a. in das Großherzogtum Luxemburg kommen, welches die ökonomisch stärkste Teilregion innerhalb der Großregion SaarLorLux darstellt. Bereits historisch dienten verschiedene Wirtschaftszweige (u.a. Montanindustrie, Glas- und Kristallindustrie) und dadurch entstandene Formen der Wirtschaftskultur als Motoren des grenzüberschreitenden Zusammenwachsens der Großregion SaarLorLux oder ihrer Teilregionen. Hier spielen die Lebenswirklichkeiten und die Alltagspraktiken der in der Großregion SaarLorLux agierenden Individuen eine wichtige Rolle. Sie zeigen, dass gerade die ökonomische Vielfalt der Teilregionen Grenzüberschreitungen befördert (z. B. Arbeitsmarktsituation, Preisniveaus von Versorgungsgütern, Immobilienmärkte). Neben diesen stark ökonomisch motivierten Grenzüberwindungen gibt es auf vielen anderen Gebieten (z. B. in Politik oder Kultur) Meilensteine des Zusammenwachsens, wie dieser Band in seiner Vielfalt gezeigt hat.

Die Großregion SaarLorLux ist schließlich v. a. eine (sozial) konstruierte, dynamische und stets kontextbezogene > Wirklichkeit < multipler Grenzziehungen . Alte Grenzen, neue Grenzen, offene Grenzen, geschlossene Grenzen, mentale Grenzen, sprachliche Grenzen, kulturelle Grenzen, ökonomische Grenzen, materielle Grenzen usw. sind letztendlich generell als (sozial) konstruiert, dynamisch und kontextbezogen anzunehmen. Damit eröffnet sich die Möglichkeit, die auch in diesem Band vorgestellten Modelle, Erfahrungen und Praktiken grenzüberschreitender Lebenswirklichkeiten und politischer Konstruktionen auf andere Grenzräume in Europa zu übertragen. Ob jedoch die Großregion SaarLorLux mit ihren Besonderheiten - etwa mit einem wirtschaftlich boomenden Nationalstaat im Zentrum oder mit vielzähligen unterschiedlich mit politischer Macht ausgestatteten Regionen - als spezifischer Einzelfall oder als Laboratorium bzw. Modellregion fungieren kann, werden zukünftige Entwicklungen zeigen müssen. Einen Ausblick darauf gibt die Frage, inwiefern die aktuellen administrativen Perimeter der Großregion SaarLorLux und die Integration der verschiedenen Teilregionen zukünftig Bestand haben werden, müssen sie sich doch der in Frankreich geplanten Reform der Regionszuschnitte stellen (Zusammenlegung Lothringens mit der Region Champagne-Ardennes und der Region Alsace) sowie den Überlegungen der amtierenden wallonischen Präsidentschaft zur Einbeziehung der Region Bruxelles-Capitale in die Großregion SaarLorLux (vgl. Clément in diesem Band). Inwiefern eine solche Großregion+ oder Megaregion SaarLorLux unterschiedliche Integrationsgrade befördert oder eine Neuausrichtung des politischen, wirtschaftlichen, kulturellen bzw. sozialen Denkens und Handelns erfordert, bleibt abzuwarten. Sollte eine solche Megaregion tatsächlich entstehen, wäre es Aufgabe zukünftiger Forschungen, die hier 
wiederum entstehenden Lebenswirklichkeiten und politischen Konstruktionen zu untersuchen. 